\title{
The assessment of intraoperative technique-related risk factors and the treatment of anastomotic leakage after esophagectomy: a narrative review
}

\author{
Chuangui Chen, Hongjing Jiang^
}

Department of Minimally Invasive Esophagus Surgery, Key Laboratory of Prevention and Therapy, National Clinical Research Center of Cancer, Tianjin Medical University Cancer Institute and Hospital, Tianjin, China

Contributions: (I) Conception and design: C Chen; (II) Administrative support: C Chen; (III) Provision of study materials or patients: C Chen; (IV) Collection and assembly of data: C Chen; (V) Data analysis and interpretation: H Jiang; (VI) Manuscript writing: All authors; (VII) Final approval of manuscript: All authors.

Correspondence to: Hongjing Jiang. Department of Minimally Invasive Esophagus Surgery, Key Laboratory of Prevention and Therapy, National Clinical Research Center of Cancer, Tianjin Medical University Cancer Institute and Hospital, Tianjin, China. Email: jianghjsci@163.com.

\begin{abstract}
Anastomotic leakage, including cervical anastomotic leakage and intrathoracic anastomotic leakage, is a serious complication of esophageal cancer and a leading cause of death after esophagectomy. In fact, anastomotic leakage after esophagectomy can be caused by numerous factors in the preoperative, intraoperative, and postoperative periods. Intraoperative technique-related risk factors, including surgical methods, anastomosis sites, anastomosis methods, the type of gastric tube, and reconstruction routes, are the key causes of its occurrence. Anastomotic leakage treatments include both surgical and non-surgical treatments, while surgical treatment has high risks, many complications, and high mortality. Actually, nonsurgical methods including naso-leakage drainage, stent, negative pressure therapy, and so on, are also very critical in the treatment of anastomotic leakage. So, the selection of correct and appropriate treatment methods plays an important role in alleviating the suffering of patients, shortening hospitalization time, and reducing mortality. This study undertook a systematic review in which data in the PubMed database were searched and analyzed to assess the safety and efficacy of surgical technique-related factors in esophagectomy, and appropriate treatment of anastomotic leakage after esophagectomy. In conclusion, gastric tube, posterior mediastinal route and stapled anastomosis are safe among esophagectomy surgical techniques, and nonsurgical treatment of anastomotic leakage such as naso-leakage drainage is feasible in the majority of cases.
\end{abstract}

Keywords: Esophageal cancer; anastomotic leakage; esophagectomy; risk factor; treatment

Submitted Dec 23, 2020. Accepted for publication Feb 08, 2021.

doi: 10.21037/jgo-21-45

View this article at: http://dx.doi.org/10.21037/jgo-21-45

\section{Introduction}

Esophageal cancer is a highly aggressive digestive tract tumor. Due to early lymph node metastasis and distant organ metastasis, it has a poor prognosis. Esophagectomy is a curative treatment for esophageal cancer; however, it is associated with significant morbidity and mortality risks. Anastomotic leakage, including cervical anastomotic leakage and intrathoracic anastomotic leakage, is one of the most serious complications of esophageal cancer and a leading cause of death (1). Additionally, anastomotic leakage can increase the risk of the local recurrence of tumors, and severe esophageal anastomotic leakage has

^ ORCID: 0000-0002-6053-283X. 
an adverse effect on tumor prognosis (2). The incidence of anastomotic leakage after esophagectomy has been reported to range from $3 \%$ to $21 \%$, and the mortality rate from $0 \%$ to $35 \%$ (1). As for intrathoracic anastomotic leakage, if it is not diagnosed and treated immediately, the mortality rate increases. Anastomotic leakage has preoperative, intraoperative, and postoperative risk factors. The intraoperative risk factors include surgical-related techniques (3), such as the esophagectomy surgical approach (open versus mini-invasive surgery), anastomosis location (intrathoracic versus cervical), anastomosis approach (handsewn versus stapled), the type of gastric conduit (gastric-tube versus whole-stomach), and the reconstruction route (anterior versus posterior mediastinal). Surgery technique is a key risk factor of anastomotic leakage, and improvements in surgery technique can lower the incidence of anastomotic leakage (4).

After esophagectomy, anastomotic leakage is a severe complication; however, there are many methods for managing it. At present, the mortality rate of anastomotic leakage is very low. Methods for managing anastomotic leakage include surgical and non-surgical treatments. The timely detection of anastomotic leakage and the adoption of a proper approach are keys to the successful treatment of anastomotic leakage. In relation to the non-surgical treatment of intrathoracic anastomotic leakage, drainage, especially naso-leakage drainage, plays an important role, because naso-leakage drainage results in minimal trauma and has a good tolerance. This review analyzed and assessed the intraoperative surgical technique-related risk factors of anastomotic leakage and a number of proper treatment methods. The ultimate goal was to provide guidance in the prevention of anastomotic leakage, and reduce the mortality rate of patients who have undergone esophagectomy. We present the following article in accordance with the Narrative Review reporting checklist (available at http:// dx.doi.org/10.21037/jgo-21-45).

\section{Literature search}

In this review, the PubMed database was searched. To identify surgical technique-related risk factors of anastomotic leakage, we included the following terms in our search: "open versus minimally invasive," "cervical versus intrathoracic," "hand-sewn versus stapled," "gastrictube versus whole-stomach," "anterior versus posterior mediastinal," "esophageal cancer," "esophagectomy," and "anastomotic leakage." We also included the synonyms and abbreviations of these terms in our search. In relation to the management of anastomotic leakage, we included the following terms in our search: "surgical treatment," "nonsurgical treatment," "drainage," "stent," "negative pressure therapy," and their synonyms and abbreviations. We analyzed sufficient and comprehensive literatures to avoid errors caused by insufficient data.

\section{Surgical methods: minimally invasive esophagectomy (MIE) versus open esophagectomy}

MIEs include the da Vinci robot-assisted minimally invasive esophagectomy (RAMIE) and the video-assisted minimally invasive esophagectomy (VAMIE). In terms of the complications that arise following these procedures, no differences between the two types of esophagectomy have been reported $(\mathrm{P}>0.05)(5,6)$. However, the question as to whether an open esophagectomy (OE) or a MIE affects anastomotic leakage after esophagectomy needs to be examined. Zhou et al. assessed the superiority of MIE over $\mathrm{OE}$ in relation to the occurrence of anastomotic leakage. Specifically, Zhou et al. conducted a meta-analysis of 43 studies involving 5,537 patients, of whom 2,527 (45.6\%) had undergone MIEs and 3,010 (54.4\%) OEs (7). The results showed that there was no statistically significant reduction in the incidence of anastomotic leakage between patients who underwent MIEs and those who underwent OEs [odds ratio $(\mathrm{OR})=0.97,95 \%$ confidence interval $(\mathrm{CI})$ $=0.80-1.17]$ (7). Zhou et al. also undertook a meta-analysis of 48 studies of 14,311 patients with esophageal cancer, and found that there was no significant difference in the occurrence of anastomotic leakage between patients who underwent MIEs and those who underwent $\mathrm{OEs}(\mathrm{OR}=0.93$, $95 \%$ CI $=0.78-1.11)(8)$. We analyzed the effects of OEs and MIEs on anastomotic leakage after esophagectomy in papers published between 2011 to 2019 as identified by our PubMed database search (see Table 1). One in eight articles reported that MIE increased the incidence of anastomotic leakage; however, the differences between $\mathrm{OE}$ and MIE were not significant $(\mathrm{OR}=0.94,95 \% \mathrm{CI}=0.88-1.21)(9-16)$. Thus, there was no evidence to suggest that MIEs are more likely to reduce the incidence of anastomotic leakages after esophagectomy than OEs.

\section{Anastomosis sites: cervical versus intrathoracic anastomosis}

The location of an esophageal tumor determines the 
Table 1 Comparison of the incidence of anastomotic leakage after esophagectomy: OE versus MIE

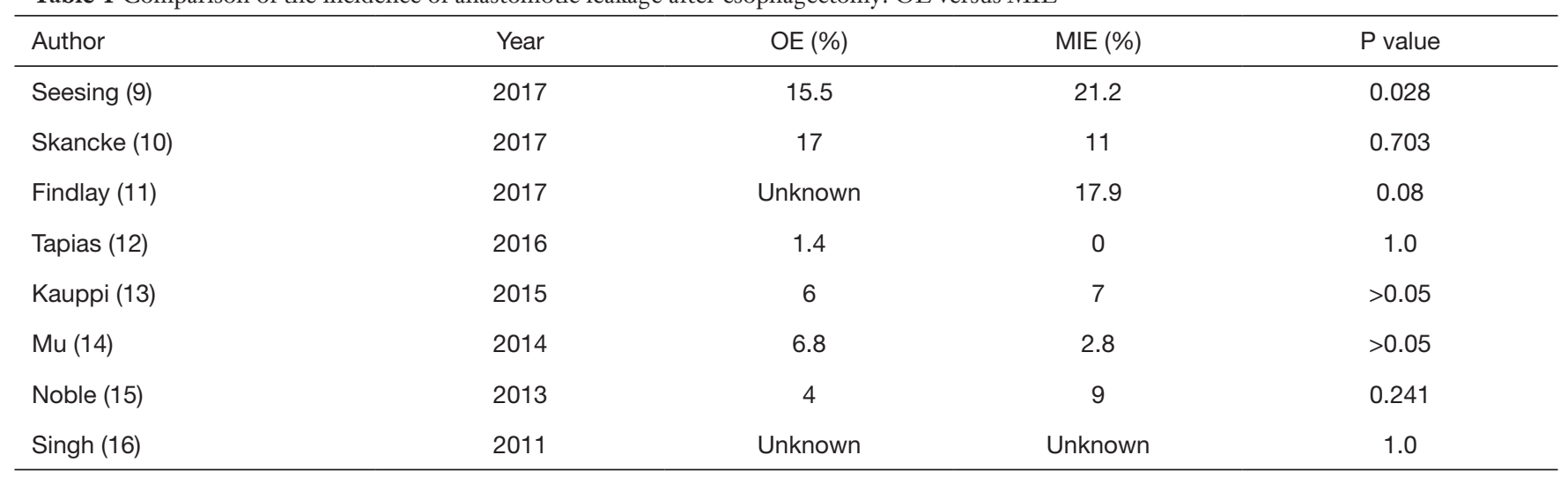

OE, open esophagectomy; MIE, minimally invasive esophagectomy.

Table 2 Comparison of the incidence of anastomotic leakage after esophagectomy: CEA versus IEA

\begin{tabular}{lcccc}
\hline Author & Year & CEA (\%) & IEA (\%) & P value \\
\hline Gooszen (17) & 2018 & 21.9 & 17.0 & 0.025 \\
Bolca (18) & 2018 & 13.8 & 1.5 & $<0.05$ \\
Huang (19) & 2015 & 8.8 & 2.2 & 0.048 \\
Zhai (20) & 2015 & 30 & 9.4 & 0.032 \\
Kassis (21) & 2013 & 12.3 & 9.3 & 0.006 \\
Klink (22) & 2012 & 31 & 11 & 0.04 \\
\hline
\end{tabular}

CEA, cervical esophagogastric anastomosis; IEA, intrathoracic esophagogastric anastomosis.

location of the esophagogastric anastomosis (EGA), which includes cervical esophagogastric anastomosis (CEA) and intrathoracic esophagogastric anastomosis (IEA). For upper thoracic and partial mid-thoracic esophageal cancer, it is more suitable to use CEA in radical resection. In our search of the PubMed database for papers published between 2011 and 2019, we included the following keywords: "cervical anastomosis," "intrathoracic anastomosis," "esophageal cancer," "esophagectomy," "anastomotic leakage," and their synonyms and abbreviations (see Table 2), and found six relevant articles. Of the six articles, all six reported that the incidence of anastomotic leakage in cervical anastomosis was higher than that in intrathoracic anastomosis (17-22). Actually, anastomosis in the cervical region must undergo a longer distance in the mediastinum and the greater tension, which will be easy to damage the periphery vascular integrity of the gastric tube, so that makes blood supply of cervical anastomosis is poorer than that of intrathoracic anastomosis. Compared to intrathoracic anastomotic leakage, cervical anastomotic leakage has a relatively low mortality rate; thus, cervical anastomoses are safer for patients (19). However, van Workum et al. reported that IEA has better functional results than CEA. Specifically, IEA has a lower benign anastomotic stricture rate and a lower incidence of recurrent laryngeal nerve palsy than CEA (23). Thus, compared with cervical anastomosis, intrathoracic anastomosis has a lower incidence of anastomotic leakage, a higher quality of life, and a higher mortality rate.

\section{Anastomosis methods: hand-sewn versus stapled anastomosis}

EGA approaches include hand-sewn (HS) and stapled anastomoses; stapled anastomoses may be either circularly stapled or linearly stapled (LS). Compared with HS anastomoses, stapled anastomoses have a lower leakage rate $(\mathrm{P}<0.05)$. As stapling produces even stitches and ensures a good blood supply, it is a relatively safe and reliable procedure (24). Deng et al.'s meta-analysis showed that the LS technique was more likely to reduce the incidence of 
anastomotic leakage and stenosis than the HS method (25). However, Markar et al. conducted a meta-analysis of 936 patients and reported that there was no significant difference between HS and stapled anastomosis cohorts (3). The difference in results might be attributable to the different methods of HS anastomoses used (i.e., singlelayer versus two-layer anastomoses). Maciver $e t$ al. reported that the two-layer anastomosis is a robust technique and has a lower leakage rate than the single-layer anastomosis technique (26). The triangulating stapled anastomoses (TSA) method has been reported to be a safe and effective alternative method for EGA, and has the advantage of a lower incidence of leakage and stenosis. The end-toend anastomosis preserves the integrity of the gastric wall vascular network and ensures additional blood supply to the anastomotic site (27). In fact, whether hand-sewn anastomosis or stapled anastomosis, even mucosa suturing and tension-free anastomosis contributes to prevent the occurrence of anastomotic leakage. Viklund reported that the risk of anastomotic leakage did not differ between HS and stapled anastomoses unless the anastomosis caused excessive tension (28). However, compared to a HS anastomosis, a stapled anastomosis represents a safer and simpler method, and is recommended for surgeons at lowvolume institutions (i.e., institutions at which $<5$ operations per year are performed) (28).

\section{Types of gastric tube: gastric tube versus whole stomach}

Compared to the whole-stomach technique, esophageal reconstruction with a gastric tube reduces the incidence of anastomotic leakage, the manifestation of intrathoracic syndrome, and the occurrence of reflux esophagitis $(22,29)$. Shu et al. reported that the probability of anastomotic leakage in the tubular stomach is lower than that in the whole stomach in three-field esophagectomy (30). Both gastric acid and anastomotic tension are important factors in the occurrence of anastomotic leakage. The more gastric tissue removed from the gastric tube, the less stomach acid secreted and the lower the risk of anastomotic leakage. As the area of the gastric tube is less than half of the gastric tissue, its area for acid secretion is also less. Further, as a result of the gastric tube, the lesser curvature and the greater curvature of the reconstructed stomach have the same length, and the anastomosis and pylorus are placed in a straight line, which solves the problems of anatomical and mechanical gastric retention and emptying problems, and thus, lowers the anastomotic tension and reduces the risk of anastomotic leakage (31). Conversely, the procedure can easily result in anastomotic leakage due to increased gastric acid secretion, greater anastomotic tension, and poorer blood supply to the stomach bottom. Indeed, the distal fundus in the gastric tube with worse circulation should be resected, which makes the anastomosis closer to the start of the right gastroepiploic artery. Further, in relation to the width of the gastric tube, it is more appropriate to create a 2-4-cm wide gastric tube, as a too-narrow tube increases the risk of gastric-tube ischemia (32).

\section{Reconstruction route: anterior versus posterior- mediastinal reconstruction}

Gastric-tube reconstruction routes have important effects on anastomotic leakage after esophagectomy. To minimize the irradiation of the gastric tube, the anterior (retrosternal) route is a better choice for esophageal reconstruction. However, compared with posterior-mediastinal reconstruction, it has a longer route and a higher incidence of cervical anastomotic leakage, which have hindered its widespread use (33). Bartels et al. strongly recommended the posterior-mediastinal route in 1993, particularly for patients with poor cardiopulmonary function (34). Fang et al. also reported that the leakage rate in a retrosternal route group (19.4\%) was significantly higher than that in a posterior-mediastinal route group $(11.9 \%, \mathrm{P}<0.05)(35)$. Due to the two angulations that are used to locate the gastric tube under xiphoid and at the inlet of the thoracic cavity from the abdomen to the neck, gastric-tube reconstruction in the retrosternal routeis more difficult to perform. The gastric tube is more easily damaged through the retrosternal route, and the retrosternal route affects the blood supply of the gastric tube, which can lead to gastrictube ischemia. Thus, anterior mediastinal reconstruction is not recommended for surgeons at low-volume institutions. However, Urschel et al. reported that the modifications of retrosternal reconstruction, such as expanding the retrosternal tunnel, widening the gastric tube, resecting the sternothyroid muscle, and the fixation of the gastric tube, decreased the incidence of cervical anastomotic leakage (36).

\section{The management of anastomotic leakage after esophagectomy}

Anastomotic leakage is defined as a disruption of the EGA, it is graded from I-IV (24) and can be divided into 
the following three stages: (I) early leakage (which occurs 1-3 days after esophagectomy); (II) mid-term leakage (which occurs 4-14 days after esophagectomy); and (III) late leakage (which occurs $>14$ days after esophagectomy). If anastomotic leakage cannot be identified and treated in time, it may endanger the patient's life. In fact, upper gastrointestinal radiography using diatrizoate meglumine is an important method for the diagnosis of anastomotic leakage after esophagectomy. Anastomotic leakage treatments may be surgical or non-surgical. Surgical anastomotic leakage treatments are associated with greater trauma, more complications, and a higher mortality rate than non-surgical anastomotic leakage treatments. Thus, non-surgical methods play an important role in the treatment of anastomotic leakage. Currently, the major methods of non-surgical treatment are drainage, stent implantation, and negative pressure therapy. However, appropriate and optimal management is the key to improving cure rates and reducing mortality rates.

\section{Surgical treatment}

In relation to cervical anastomotic leakages, it is simpler to open a cervical wound at the bedside and initiate twice-daily dressing changes. Additionally, anastomotic leakages heal more easily. Conversely, intrathoracic leakages, including intrathoracic manifestations of cervical anastomotic leakages, are more complex and difficult to manage. Due to greater trauma and more complications, we usually do not recommend that intrathoracic anastomotic leakages be treated with surgery. In our experience, the early surgical repair of anastomotic leakages is effective in treating early leakage. In relation to mid-term or late leakage, surgical repair is not recommended. In respect of major intrathoracic leakages without serious pleural adhesion, thoracoscopic explorations can improve drainage and eliminate cellulose on the trapped lung surface, which can promote lung recruitment. In relation to major leakages with serious pleural adhesion, open thoracic exploration is required in selected cases, which can improve drainage, clean the separated abscess and promote lung recruitment. On occasion, we used omentum or muscle flaps in large anastomotic leakages or staple line defects, but the likelihood of success is limited. Reconstructions can be performed using colon and retrosternal routes to treat some late leakages; however, this increases the mortality rate. Thus, for patients with anastomotic leakages or tube necrosis-required reoperations, non-surgical treatments have been shown to be feasible in the majority of cases (37).

\section{Non-surgical treatment: naso-leakage drainage}

Intrathoracic anastomotic leakage after esophagectomy is accompanied by high morbidity and mortality. Indeed, the intrathoracic manifestation of cervical anastomotic leakage occurs in more than $50 \%$ of patients and immediate and sufficient drainage is required to prevent further contamination of the content of the gastrointestinal tract (38). Drainage includes naso-leakage drainage and pleural drainage. In circumstances in which percutaneous abscess drainage is technically impossible or ineffective, nasoleakage drainage plays an important role. A naso-leakage tube is placed through the anastomotic leakage at the bottom of the abscess cavity under the guidance of an ultraslim electronic gastroscope or using the interventional radiology technique. The naso-leakage tube is connected to a negative vacuum device for drainage and irrigation. When the abscess cavity diminishes, the drainage appears clean, and after confirmation that the leakage is healing through upper gastrointestinal radiography using diatrizoate meglumine, the naso-leakage tube can be gradually removed. Shuto et al. examined 25 patients who underwent naso-esophageal extraluminal drainage accompanied with enteral nutrition. Of the patients, $21(84 \%)$ patients had major leakages, 1 (4\%) had a minor leakage, and $3(12 \%)$ had tube necrosis. None of the naso-esophageal drainage cases $(100 \%)$ required reoperation or reintervention, and all patients were completely cured (100\%) during hospitalization without any death (39).

In addition to being minimally invasive, naso-esophageal drainage and concomitant enteral nutritional support have been shown to be effective and reliable methods for treating major leakages after esophagectomy $(39,40)$. In our experience, adequate drainage is key to the successful treatment of anastomotic leakages, while naso-leakage extraluminal drainage with or without pleural drainage combined with fasting, water, antibiotics, and enteral nutrition is safe and effective. From 2010 to the present, all patients with anastomotic leakages after esophagectomy have been cured with naso-leakage extraluminal drainage, and the mortality rate has been 0 . Figure 1 shows a major leakage, by interventional radiology, whereby a nasoleakage tube was placed into the abscess cavity through the leakage. After three months of naso-leakage drainage, the anastomotic leakage healed successfully. 

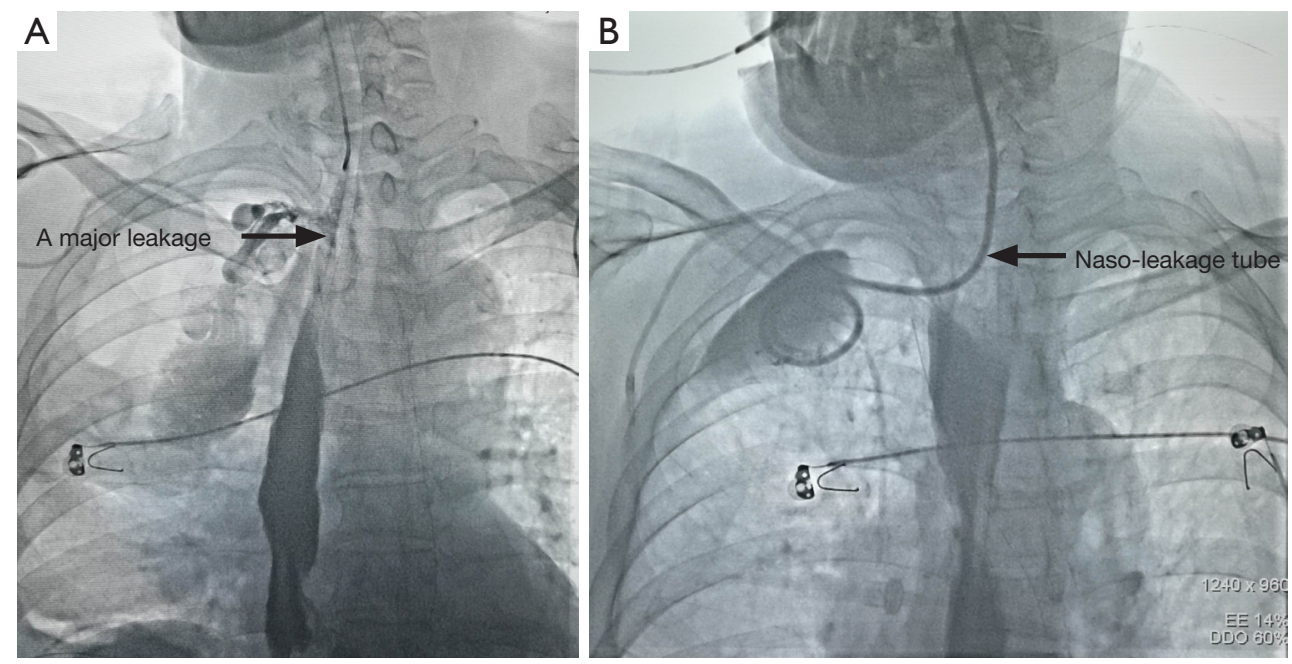

Figure 1 Naso-leakage drainage. (A) An interventional radiology procedure showed a major leakage at the esophagogastric anastomosis; (B) a naso-leakage tube was placed into the abscess cavity through the leakage.

\section{Non-surgical treatment: stent}

Stent implantation is another method of non-surgical treatment. It can significantly reduce the morbidity and mortality associated with intrathoracic leakages (but not cervical leakages) after esophagectomy. Cervical leakages can be treated by opening the wound in the neck; however, the intrathoracic manifestation of cervical anastomotic leakages requires further treatment. Types of stents include partially and fully covered self-expanding metal stents (SEMS) and self-expanding plastic stents (SEPS). Stents are used to diminish the extra-anastomotic contamination of the pleura and mediastinum and promote leakage healing. However, the application of a stent may be accompanied by some complications, including bleeding, stent migration, leakage deterioration, tissue ingrowth, gastric-tube wall perforation, and death from aorto esophageal fistula. Schweigert et al. reported that endoscopic stent implantation increased the incidence of postoperative aorto esophageal fistulas due to the stent-related erosion of the thoracic aorta, and that the mean time of aorto esophageal fistula occurrence after stent insertion was approximately 26 days (41). Thus, the risk of life-threatening complications after stent implantation needs to be acknowledged (41).

Dasari et al. reported that endoscopic stent treatment is safe and effective for esophageal anastomotic leakages and perforations accompanied by adequate pleural and mediastinal drainage (42). Thus, stent applications are usually limited to patients with $30 \%$ leakages around the esophageal circumference and without extensive necrosis of the gastric tube. Further, stent applications should be used in combination with drainage to prevent the aggravation of the infection and anastomotic tissue necrosis. If a stent treatment does not lead to any clinical improvement, the removal of the stent or surgical re-exploration should be considered.

\section{Non-surgical treatment: negative pressure therapy}

Recently, endoscopic vacuum-assisted closure (E-VAC) therapy was introduced as a novel treatment for anastomotic leakages after esophagectomy; however, it has not been widely used due to a lack of corresponding equipment and experience $(43,44)$. We conducted a search for a number of terms, including "esophagus," "esophageal," "esophagectomy," "anastomotic leakage," "endoscopic VAC," "negative pressure therapy", in the PubMed database, and found that only a few articles on E-VAC have been published. In a VAC acts, a vacuum device is applied to the wound via a vacuum-sealed sponge. The vacuum drainage can be positioned through the defect into the extraluminal wound cavity. The intraluminal vacuum drainage is connected to an electronically controlled vacuum device, and a continuous negative pressure of $100-125 \mathrm{mmHg}$ is maintained for several days. The esophageal lumen or wound cavity then collapses around the drainage, resulting in an intraluminal evacuation and the closure of the defect. The sponge continuously removes wound secretions 
and interstitial edemas, improves microcirculation, promotes granulation tissue formation, and thus results in the healing of leakage. E-VAC is an effective treatment method for intrathoracic anastomotic leakages and has been shown to be more effective than stent implantation $(45,46)$. The combination of E-VAC and stent application represents a successful and novel method for improving the healing rates of intrathoracic anastomotic leakages (47). However, E-VAC is more suitable for early and relatively small anastomotic leakages and perforations (48). Further, it should be noted that the efficacy of E-VAC in treating anastomotic leakages requires further study.

\section{Summary}

There are many risk factors related to intraoperative techniques, such as the whole-stomach technique, anterior mediastinal route, and cervical anastomosis, which are associated with the incidence of anastomotic leakage after esophagectomy, while the site of anastomosis is plays an important role in affecting anastomotic leakage. The better esophagectomy surgical techniques to reduce anastomotic leakage appear to be the gastric tube, posterior-mediastinal route, and stapled anastomoses. In relation to the treatment of anastomotic leakages, different treatment methods should be selected according to the anastomotic leakage stage and grade. As far as possible, non-surgical treatments such as naso-leakage drainage, which cause less trauma and fewer complications, should be adopted.

\section{Acknowledgements}

Funding: The National Key Clinical Specialist Construction Programs of China (No. 2013-544) financed this study.

\section{Footnote}

Reporting Checklist: The authors have completed the Narrative Review reporting checklist. Available at http:// dx.doi.org/10.21037/jgo-21-45

Conflicts of Interest: All authors have completed the ICMJE uniform disclosure form (available at http://dx.doi. org/10.21037/jgo-21-45). The authors have no conflicts of interest to declare.

Ethical Statement: The authors are accountable for all aspects of the work in ensuring that questions related to the accuracy or integrity of any part of the work are appropriately investigated and resolved.

Open Access Statement: This is an Open Access article distributed in accordance with the Creative Commons Attribution-NonCommercial-NoDerivs 4.0 International License (CC BY-NC-ND 4.0), which permits the noncommercial replication and distribution of the article with the strict proviso that no changes or edits are made and the original work is properly cited (including links to both the formal publication through the relevant DOI and the license). See: https://creativecommons.org/licenses/by-nc-nd/4.0/.

\section{References}

1. Low DE. Diagnosis and management of anastomotic leaks after esophagectomy. J Gastrointest Surg 2011;15:1319-22.

2. Markar S, Gronnier C, Duhamel A, et al. The impact of severe anastomotic leak on long-term survival and cancer recurrence after surgical resection for esophageal malignancy. Ann Surg 2015;262:972-80.

3. Markar SR, Arya S, Karthikesalingam A, et al. Technical factors that affect anastomotic integrity following esophagectomy: systematic review and meta-analysis. Ann Surg Oncol 2013;20:4274-81.

4. $\mathrm{Ng}$ T, Vezeridis MP. Advances in the surgical treatment of esophageal cancer. J Surg Oncol 2010;101:725-9.

5. Yerokun BA, Sun Z, Yang CJ, et al. Minimally invasive versus open esophagectomy for esophageal cancer: apopulation-based analysis. Ann Thorac Surg 2016;102:416-23.

6. Nakauchi M, Uyama I, Suda K, et al. Robotic surgery for the upper gastrointestinal tract: Current status and future perspectives. Asian J Endosc Surg 2017;10:354-63.

7. Zhou C, Ma G, Li X, et al. Is minimally invasive esophagectomy effective for preventing anastomotic leakages after esophagectomy for cancer? A systematic review and meta-analysis. World J Surg Oncol 2015;13:269.

8. Zhou C, Zhang L, Wang H, et al. Superiority of minimally invasive oesophagectomy in reducing in-hospital mortality of patients with resectable oesophageal cancer: a metaanalysis. PLoS One 2015;10:e0132889.

9. Seesing MFJ, Gisbertz SS, Goense L, et al. A propensity score matched analysis of open versus minimally invasive transthoracic esophagectomy in the Netherlands. Ann Surg 2017;266:839-46.

10. Skancke MD, Grossman RA, Marino G, et al. Analysis 
of minimally invasive esophagectomy at a single Veterans Affairs Medical Center. J Laparoendosc Adv Surg Tech A 2017;27:784-9.

11. Findlay L, Yao C, Bennett DH, et al. Non-inferiority of minimally invasive oesophagectomy: an 8-year retrospective case series. Surg Endosc 2017;31:3681-9.

12. Tapias LF, Mathisen DJ, Wright CD, et al. Outcomes with open and minimally invasive Ivor Lewis esophagectomy after neoadjuvant therapy. Ann Thorac Surg 2016;101:1097-103.

13. Kauppi J, Räsänen J, Sihvo E, et al. Open versus minimally invasive esophagectomy: clinical outcomes for locally advanced esophageal adenocarcinoma. Surg Endosc 2015;29:2614-9.

14. Mu J, Yuan Z, Zhang B, et al. Comparative study of minimally invasive versus open esophagectomy for esophageal cancer in a single cancer center. Chin Med J (Engl) 2014;127:747-52.

15. Noble F, Kelly JJ, Bailey IS, et al. A prospective comparison of totally minimally invasive versus open Ivor Lewis esophagectomy. Dis Esophagus 2013;26:263-71.

16. Singh RK, Pham TH, Diggs BS, et al. Minimally invasive esophagectomy provides equivalent oncologic outcomes to open esophagectomy for locally advanced (stage II or III) esophageal carcinoma. Arch Surg 2011;146:711-4.

17. Gooszen JAH, Goense L, Gisbertz SS, et al. Intrathoracic versus cervical anastomosis and predictors of anastomotic leakage after oesophagectomy for cancer. Br J Surg 2018;105:552-60.

18. Bolca C, Dumitrescu M, Fotache G, et al. Comparative study of early postoperative complications: thoracic anastomosis vs cervical anastomosis - in esophageal replacement with gastric graft. Chirurgia (Bucur) 2018;113:95-100.

19. Huang HT, Wang F, Shen L, et al. Clinical outcome of middle thoracic esophageal cancer with intrathoracic or cervical anastomosis. Thorac Cardiovasc Surg 2015;63:328-34.

20. Zhai C, Liu Y, Li W, et al. A comparison of short-term outcomes between Ivor-Lewis and McKeown minimally invasive esophagectomy. J Thorac Dis 2015;7:2352-8.

21. Kassis ES, Kosinski AS, Ross P Jr, et al. Predictors of anastomotic leak after esophagectomy: an analysis of the society of thoracic surgeons general thoracic database. Ann Thorac Surg 2013;96:1919-26.

22. Klink CD, Binnebösel M, Otto J, et al. Intrathoracic versus cervical anastomosis after resection of esophageal cancer: a matched pair analysis of 72 patients in a single center study. World J Surg Oncol 2012;10:159.

23. van Workum F, van der Maas J, van den Wildenberg FJ, et al. Improved functional results after minimally invasive esophagectomy: intrathoracic versus cervical anastomosis. Ann Thorac Surg 2017;103:267-73.

24. Price TN, Nichols FC, Harmsen WS, et al. A comprehensive review of anastomotic technique in 432 esophagectomies. Ann Thorac Surg 2013;95:1154-60.

25. Deng XF, Liu QX, Zhou D, et al. Hand-sewn vs linearly stapled esophagogastric anastomosis for esophageal cancer: a meta-analysis. World J Gastroenterol 2015;21:4757-64.

26. Maciver RH, Sundaresan S, DeHoyos AL, et al. Mucosal tube technique for creation of esophageal anastomosis after esophagectomy. Ann Thorac Surg 2009;87:1703-7.

27. Li J, Shen Y, Tan L, et al. Cervical triangulating stapled anastomosis: technique and initial experience. J Thorac Dis 2014;6 Suppl 3:S350-4.

28. Viklund P, Lindblad M, Lu M, et al. Risk factors for complications after esophageal cancer resection: a prospective population-based study in Sweden. Ann Surg 2006;243:204-11.

29. Zhang W, Yu D, Peng J, et al. Gastric-tube versus whole-stomach esophagectomy for esophageal cancer: A systematic review and meta-analysis. PLoS One 2017;12:e0173416.

30. Shu YS, Sun C, Shi WP, et al. Tubular stomach or whole stomach for esophagectomy through cervico-thoracoabdominal approach: a comparative clinical study on anastomotic leakage. Ir J Med Sci 2013;182:477-80.

31. Chen KN. Managing complications I: leaks, strictures, emptying, reflux, chylothorax. J Thorac Dis 2014;6 Suppl3:S355-63.

32. He X, Shi M, Cao B. Impact of gastric tube diameter on quality of life of esophagus cancer patient after Ivor-Lewis esophagectomy. Zhonghua Wei Chang Wai Ke Za Zhi 2018;21:1001-7.

33. Coral RP, Constant-Neto M, Silva IS, et al. Comparative anatomical study of the anterior and posterior mediastinum as access routes after esophagectomy. Dis Esophagus 2003;16:236-8.

34. Bartels H, Thorban S, Siewert JR. Anterior versus posterior reconstruction after transhiatal oesophagectomy: a randomized controlled trial. Br J Surg 1993;80:1141-4.

35. Fang WT, Chen WH, Fan LM, et al. Causes and prevention of anastomotic leakage after esophagectomy and reconstruction through different routes for esophageal cancer. Zhonghua Wei Chang Wai Ke Za Zhi 2005;8:217-9. 36. Urschel JD, Urschel DM, Miller JD, et al. A meta-analysis 
of randomized controlled trials of route of reconstruction after esophagectomy for cancer. Am J Surg 2001;182:470-5.

37. Sarli D, Bona D, Abraham M, et al. Conservative and surgical treatment of esophago-gastric anastomotic leaks. Ann Ital Chir 2006;77:391-6.

38. van Rossum PS, Haverkamp L, Carvello M, et al. Management and outcome of cervical versus intrathoracic manifestation of cervical anastomotic leakage after transthoracic esophagectomy for cancer. Dis Esophagus 2017;30:1-8.

39. Shuto K, Kono T, Akutsu Y, et al. Naso-esophageal extraluminal drainage for postoperative anastomotic leak after thoracic esophagectomy for patients with esophageal cancer. Dis Esophagus 2017;30:1-9.

40. Zhang Y, Zhang YX, Hu JW, et al. Endoscopic naso-leakage drainage: a safe and effective method for the management of intrathoracic anastomotic leakage after esophagectomy. J Thorac Dis 2017;9:3052-61.

41. Schweigert M, Dubecz A, Stadlhuber RJ, et al. Risk of stent-related aortic erosion after endoscopic stent insertion for intrathoracic anastomotic leaks after esophagectomy. Ann Thorac Surg 2011;92:513-8.

42. Dasari BV, Neely D, Kennedy A, et al. The role of esophageal stents in the management of esophageal anastomotic leaks and benign esophageal perforations. Ann
Surg 2014;259:852-60.

43. de Moura DTH, de Moura BFBH, Manfredi MA, et al. Role of endoscopic vacuum therapy in the management of gastrointestinal transmural defects. World J Gastrointest Endosc 2019;11:329-44.

44. Loske G. Endoscopic Negative Pressure Therapy of the Upper Gastrointestinal Tract. Chirurg 2019;90:1-6.

45. Mennigen R, Harting C, Lindner K, et al. Comparison of endoscopic vacuum therapy versus stent for anastomotic leak after esophagectomy. J Gastrointest Surg 2015;19:1229-35.

46. Brangewitz M, Voigtländer T, Helfritz FA, et al. Endoscopic closure of esophageal intrathoracic leaks: stent versus endoscopic vacuum-assisted closure, a retrospective analysis. Endoscopy 2013;45:433-8.

47. Bludau M, Hölscher AH, Herbold T, et al. Management of upper intestinal leaks using an endoscopic vacuum-assisted closure system (E-VAC). Surg Endosc 2014;28:896-901.

48. Neumann PA, Mennigen R, Palmes D, et al. Pre-emptive endoscopic vacuum therapy for treatment of anastomotic ischemia after esophageal resections. Endoscopy 2017;49:498-503.

(English Language Editor: L. Huleatt)

Cite this article as: Chen C, Jiang H. The assessment of intraoperative technique-related risk factors and the treatment of anastomotic leakage after esophagectomy: a narrative review. J Gastrointest Oncol 2021;12(1):207-215. doi: 10.21037/jgo-21-45 